\title{
Д.А. кЛЮйко
}

\section{ПАТОГЕНЕТИЧЕСКИЕ ПРЕДПОСЫЛКИ РАЗВИТИЯ СПАЕЧНОГО ПРОЦЕССА БРЮШНОЙ ПОЛОСТИ}

\author{
Белорусский государственный медицинский университет, г. Минск, \\ Республика Беларусь
}

В литературе ведется дискуссия, касающаяся профилактики, диагностики и лечения спаечной болезни брюшной полости и ассоциированной с ней кишечной непроходимости, при этом совершенно упускается из виду вопрос патогенеза заболевания и истинных причин его развития. За последнее время не было существенных достижений в решении данной проблемы. Анализ литературных источников выявил ряд недостатков научно-практического подхода, связанных с устаревшими фундаментальными представлениями о причинах спаечного процесса, основу которого составляют: оксигенация тканей, организация фибрина. Так, заживление брюшины и восстановление нативного слоя мезотелиоцитов без образования спаек возможно лишь в первые 3 дня с момента повреждения. При этом важнейшую роль в формировании спаек брюшины играет гипоксия, влияя на созревание мезотелиоцитов и дифференцировку фибробластов. Решение проблемы спайкообразования возможно посредством воздействия на ключевые звенья патогенеза: гипоксию тканей брюшной полости, систему деградации фибринолитического и внеклеточного матрикса, образование и активацию аутокоидов и регуляторов тканевого фиброза. Невзирая на традиционно сложившиеся подходы необходим пересмотр концепции патогенеза данного заболевания с точки зрения молекулярно-клеточных механизмов образования спаек.

Ключевые слова: брюшная полость, осложнения, общая хирургия, кишечная непроходимость, адгезия тканей, TGF- $\beta, H I F$.

In the literature, there is a discussion concerning the prevention, diagnosis and treatment of adhesions of the abdominal cavity and associated with intestinal obstruction, while the question of the pathogenesis of the disease and the true reasons for its development is completely overlooked. Recently, there have been no any significant achievements in solving this problem. The analysis of literature sources revealed a number of shortcomings of the scientific and practical approach associated with outdated fundamental ideas about the causes of the adhesive process, which are based on tissue oxygenation and fibrin organization. So, the healing of the peritoneum and the restoration of the native layer of mesioteliocetes without the formation of adhesions is possible only in the first 3 days from the moment of an injury. At the same time, hypoxia plays an important role in the formation of peritoneal adhesions, affecting the maturation of mesothelial cells and the differentiation of fibroblasts. The solution to the problem of adhesion is possible by influencing the key links of pathogenesis: hypoxia in the abdominal tissue, the system of fibrinolytic and extracellular matrix degradation, the formation and activation of autocoids and regulators of fibrotic tissue. Despite the traditionally established approaches, it is necessary to revise the concept of the pathogenicity theory understanding the cellular and molecular mechanisms of adhesion formation .

Keywords: abdominal cavity, complications, general surgery, intestinal obstruction, tissue adhesion, TGF- $\beta$, HIF

Novosti Khirurgii. 2021 Nov-Dec; Vol 29 (6): 728-735

The articles published under CC BY NC-ND license

Pathogenetic Prerequisites for the Development of the Adhesion Process of the Abdominal Cavity D.A. Klyuiko

\section{Введение}

Внутрибрюшные спайки возникают у 93\% пациентов после абдоминальных операций, вызывая непроходимость тонкой кишки, бесплодие, хронические боли в животе, и увеличивают вероятность ятрогенного повреждения кишечника при повторной операции [1, 2, 3]. До $30 \%$ ранее оперированных пациентов нуждаются в серии повторных вмешательств, направленных на устранение спаечного процесса, при этом в $15 \%$ случаев применяется лапароскопический адгезиолизис [2, 4, 5].

Спаечный процесс в брюшной полости
(БП) развивается в результате механической травмы, воспаления и коагуляции, которые повреждают клеточный монослой, расположенный на базальной мембране, оставляя ее открытой для отложения фибрина, что способствует дальнейшему прикреплению фибробластов и образованию сосудистой сети $[6,7,8]$. Сопутствующее снижение фибринолитической активности приводит к формированию внеклеточного матрикса и образованию адгезии [9].

В зависимости от своего местоположения, структуры и производной патологии спайки могут оставаться незаметными или вызывать жизнеугрожающие осложнения [10]. Абдоми- 
нальные спайки приводят к боли в животе, непроходимости тонкой кишки, женскому бесплодию, существенно снижая качество жизни пациентов [5, 11]. Таким образом, разработка профилактических мер, предотвращающих образование спаек, имеет решающее значение для улучшения результатов хирургического вмешательства, снижения боли, частоты повторных операций и последующих сопутствующих экономических затрат [5].

За последнее время не было существенных достижений в решении данной проблемы. Спаечный процесс обычно недооценивается хирургами как на уровне отдельного пациента, так и в масштабе проблемы практической хирургии в целом $[5,14,15]$.

Таким образом, необходимы дальнейшие усилия в разработке эффективной системы, которая предотвратит образование послеоперационных спаек.

\section{Материал и методы}

По определению спаечная болезнь представляет собой отдельную нозологическую форму с рядом характерных черт, а именно: образованием сращений брюшины, ассоциированных с кишечной непроходимостью и/или болевым синдромом [16].

С целью сбора и систематизации литературных данных нами проведен поиск в публикациях и систематических обзорах, результатов рандомизированных клинических исследований. С использованием комбинации поисковых запросов «послеоперационные спайки», «спаечная болезнь брюшной полости», «профилактика», «лечение» отобраны научные и оригинальные статьи, опубликованные между 2010 и 2021 годами. Поиск проводили с помощью систем PubMed, Scopus, Google Scholar, Elibrary на английском или русском языках.

\section{Обсуждение}

Проведенный анализ литературных источников выявил достаточно весомое несоответствие в терминологии. Так, например, при выполнении операции пациенту устанавливается диагноз «острая спаечная кишечная непроходимость» а при успешном консервативном лечении, зачастую пациенты выписываются с диагнозом «спаечная болезнь».

Симонян К.С. определяет спаечную болезнь как заболевание, в основе патогенеза которого лежит спаечный процесс в БП [17]. Напалков П.Н. утверждает, что в развитии спаечной болезни сращения брюшины имеют второстепенное значение. Поэтому он предлагает следующее определение: «брюшинный перинеальный фиброз - очаговый или диффузный», при этом термин «хроническая болезнь» считает необоснованным [18, 19]. Ю.Л. Шальков определяет нарушение пассажа, по причине частичного или полного механического препятствия вследствие спаечного процесса как спаечный синдром [16, 20].

До сих пор не ясно, как определять место препятствия, используя неинвазивные методы исследования [21, 22]. Информативность традиционных рентгенологических методов (обзорной рентгенографии и рентгеноскопии БП) зачастую не позволяет определить локализацию препятствия. Отсутствуют критерии определения места и степени обструкции [23].

Нерешенным является вопрос о значении брюшинных спаек в развитии абдоминального болевого синдрома. Спайки считаются основой в механизме развития хронической тазовой боли, а их хирургическое удаление является методом выбора [24]. При этом не было выявлено клинически значимой разницы в локализации и плотности спаек в группах пациентов с бессимптомным бесплодием и хронической тазовой болью [22].

\section{Патогенез спайкообразования}

Гистамин и брадиинин, а также другие аутокоиды стимулируют ноцицепторы, позволяют усомниться в роли спаек таза в развитии хронической тазовой боли [25]. С другой стороны, считается, что спайки вызывают боль косвенно, ограничивая движение органов, растягивая гладкие мышцы прилегающих внутренних органов или брюшной стенки [18].

Доказано, что нервные волокна, идентифицированные гистологически, ультраструктурно и иммуногистохимически, имеют место во всех сращениях брюшины. Вне зависимости от наличия хронического абдоминального болевого синдрома, во всех фиброзных тканях спаек присутствовали сенсорные нейрональные маркеры, протеин, связанный с геном кальцитонина, и вещество Р.

Данное исследование показало, что эти структуры способны передавать боль после соответствующей стимуляции, а спайки брюшины могут быть причиной хронической боли [13].

В литературе ведется дискуссия, касающаяся профилактики, диагностики и лечения спаечной кишечной непроходимости, при этом совершенно упускается из виду вопрос патогенеза заболевания, истинных причин его развития [13].

Патогенез спайкообразования инициируется операционной травмой и воспалением 
и влечет за собой ингибирование деградации внеклеточного и фибринолитического матрик$\mathrm{ca}$, образование цитокинов, главным образом трансформирующего фактора роста-1 (TGF-1), ключевого регулятора тканевого фиброза и гипоксию тканей в результате нарушения кровоснабжения мезотелиальных клеток и субмезотелиальных фибробластов [26, 27, 28, 29].

Брюшина выстлана мезотелиоцитами, слабо фиксированными к базальной мембране, легко отсоединяемыми при воспалении или незначительной травме [30, 31, 32]. После повреждения брюшины местная воспалительная реакция вызывает повышенную проницаемость сосудов, снабжающих поврежденную область, с последующей экссудацией серозно-геморрагической жидкости, богатой фибрином и воспалительными клетками, что в конечном итоге приводит к образованию фибринового матрикса. Обычно экспрессия активатора плазминогена разрушает фибринозную массу, способствует заживлению перитонеальных поверхностей в течение 3-5 дней без спаек. Однако, если экспрессия снижается, фибринозная масса сохраняется, и лежащие в основе фибробласты мигрируют в нее. Затем фибробласты формируют внеклеточный матрикс, включая коллаген и фибронектин, что приводит к образованию адгезии [33, 34, 35].

Роль фибринолиза в формировании и реформировании адгезии заключается в разрушении фибриновых сгустков, образующихся в процессе заживления. Неактивный профермент плазминоген превращается в плазмин, который разрушает фибрин и ограничивает образование адгезии.

Как механическое, так и химическое повреждение уменьшают экспрессию активатора плазминогена в первые часы после хирургической операции, за которыми следует полная потеря фибринолитической активности в течение 72 часов [36].

Во время острой фазы воспалительного ответа мезотелиальные клетки и перитонеальные макрофаги продуцируют различные цитокины, включая TGF-1, фактор некроза опухоли, интерлейкин-1 и интерлейкин-6. TGF-1 не только взаимодействует с фибринолитической системой внеклеточного матрикса, но и со многими другими клеточными медиаторами, участвующими в процессе образования адгезии. Сверхэкспрессия TGF-1 брюшиной, а также повышенные концентрации TGF-1 в перитонеальной жидкости связаны с увеличением частоты образования спаек [37, 38, 39].

Таким образом, в основе патогенеза спаечного процесса лежит организация фибринового матрикса в результате интраперитонеальной экссудации свободного фибрина [27, 28], с инициацией через 12 часов после повреждения брюшины. При разрушении фибринового матрикса в первые 72 часа, когда уровень TGF-1, ответственного за клеточный апоптоз и подавление пролиферации клеток, высок, дефект брюшины заполняется нативными мезотелиоцитами и заживление происходит без спаек. Наиболее критическими являются 5-6 сутки после повреждения по причине активизации ангиогенеза. В этот период количество макрофагов, достигнув пика, снижается, при этом большая часть поверхности уже заполнена мезотелиоцитами. Активность TGF-1, фактора роста и созревания сосудов также повышаются параллельно с воспалительным ответом, связанным с тканевым ремоделированием - основой структурной стабилизации спайки $[38,40]$.

\section{Профилактика спаечного процесса}

Основным направлением профилактики образования внутрибрюшных сращений является минимизация операционной травмы. При проведении всех абдоминальных хирургических вмешательств следует соблюдать принципы, близкие к «принципам Холстеда» (W.S. Halsted, 1852-1922), первого хирурга, осознавшего чрезвычайную важность мер профилактики.

Необходимыми условиями профилактики являются: осторожное обращение с тканями, гемостаз, непрерывная ирригация и избегание высыхания, эффективное использование инструментов, минимизация швов и компрессии тканей. Использование тонких атравматичных игл и неопудренных перчаток также способствует профилактике спайкообразования. Однако современные исследования доказывают, что инородные тела редко являются причиной образования спаек при отсутствии повреждения мезотелия брюшины [41, 42].

Экспериментально доказано, что незакрытые интраоперационные дефекты брюшины способствуют снижению образования спаек [17]. Другие авторы указывают на отсутствие клинически значимой разницы или даже на уменьшение вероятности образования спаек при ушивании таких дефектов [13].

Предполагается, что ключевую роль в формировании спаек играет гипоксия брюшины $[43,44,45]$. Во время лапаротомии повреждение ткани, включая травму, высыхание и разрушение сосудов, снижает поступление кислорода в брюшину. Было доказано, что лапароскопические операции менее адгезиогенные не только потому, что менее травматичны, но также изза повышенного давления кислорода в ткани 
брюшины по сравнению с таковым во время лапаротомии [46].

При лапароскопии СО2-пневмоперитонеум непосредственно влияет на образование спаек БП. Было продемонстрировано, что количество сращений и их плотность увеличиваются с продолжительностью СО2-пневмоперитонеума и величиной внутрибрюшного давления [10, 47, 48]. Негативное влияние CO2-пневмоперитонеума нивелируется при добавлении 2-4\% кислорода и указывает на важную роль гипоксии в образовании спаек.

Углекислый газ, используемый в лапароскопической хирургии, холодный и сухой, не является физиологическим [34, 46, 49]. Неоспоримы преимущества инсуффляции $\mathrm{CO} 2$, нагретого до $37^{\circ} \mathrm{C}$ и увлажненного (относительная влажность 95\%), способствующей меньшему охлаждению, сокращению времени пребывания в стационаре, раннему купированию послеоперационной боли и уменьшению образования адгезии [48].

\section{Заключение}

Анализ литературных источников выявил ряд недостатков научно-практического подхода, связанных с устаревшими фундаментальными представлениями о причинах спаечного процесса. Невзирая на традиционно сложившиеся подходы необходим пересмотр концепции патогенеза данного заболевания с точки зрения молекулярно-клеточных механизмов образования спаек.

Лапароскопические операции менее адгезиогенные по причине меньшей травматичности, десикации поверхности брюшины и деструкции сосудов.

Ключевую роль в формировании спаек играет гипоксия брюшины, влияя на созревание мезотелиоцитов и дифференцировку фибробластов.

\section{Финансирование}

Работа выполнялась в соответствии с планом научных исследований кафедры военно-полевой хирургии военно-медицинского факультета в УО «Белорусский государственный медицинский университет». Финансовой поддержки со стороны компаний-производителей лекарственных препаратов авторы не получали.

\section{Конфликт интересов}

Автор заявляет, что конфликт интересов отсутствует.

\section{ЛИТЕРАТУРА}

1. Bello-Guerrero JA, Cruz-Santiago CA, LunaMartínez J. Pirfenidone vs. sodium hyaluronate/ carboxymethylcellulose as prevention of the formation of intra-abdominal adhesions after colonic surgery. A randomized study in an experimental model. Cir Esp. 2016 Jan;94(1):31-37. doi: 10.1016/j.ciresp.2015.06.005 2. Beyene RT, Kavalukas SL, BarbulA. Intra-abdominal adhesions: Anatomy, physiology, pathophysiology, and treatment. Curr Probl Surg. 2015 Jul;52(7):271-19. doi: 10.1067/j.cpsurg.2015.05.001

3. Skoglar A, Gunnarsson U, Falk P. Band adhesions not related to previous abdominal surgery - A retrospective cohort analysis of risk factors. Ann Med Surg (Lond). 2018 Nov 13;36:185-90. doi: 10.1016/j. amsu.2018.11.007. eCollection 2018 Dec.

4. Uslu Yuvaci H, Cevriolu AS, Gündüz Y, Akdemir N, Karacan A, Erkorkmaz Ü, Keskin A. Does applied ultrasound prior to laparoscopy predict the existence of intra-abdominal adhesions? Turk J Med Sci. 2020 Apr 9;50(2):304-11. doi: 10.3906/sag-1910-61. Online ahead of print.

5. Луцевич ОЭ, Акимов ВП, Ширинский ВГ, Бичев АА. Вопросы патогенеза спаечной болезни брюшины и современные подходы к ее предупреждению: обзор литературы. Москов Хирург Журн. 2017;(3):1126. https://www.mossj.ru/jour/article/view/22

6. Abrahams AC, Habib SM, Dendooven A, Riser BL, van der Veer JW, Toorop RJ, Betjes MG, Verhaar MC, Watson CJ, Nguyen TQ, Boer WH. Patients with encapsulating peritoneal sclerosis have increased peritoneal expression of connective tissue growth factor (CCN2), transforming growth factor-1, and vascular endothelial growth factor. PLoS One. 2014 Nov 10;9(11):e112050. doi: 10.1371/journal.pone.0112050. eCollection 2014.

7. Midgley AC, Rogers M, Hallett MB, Clayton A, Bowen T, Phillips AO, Steadman R. Transforming growth factor-1 (TGF-1)-stimulated fibroblast to myofibroblast differentiation is mediated by hyaluronan (HA)-facilitated epidermal growth factor receptor (EGFR) and CD44 co-localization in lipid rafts. $J$ Biol Chem. 2013 May 24;288(21):14824-38. doi: 10.1074/ jbc.M113.451336

8. Zweers MM, Struijk DG, Smit W, Krediet RT. Vascular endothelial growth factor in peritoneal dialysis: a longitudinal follow-up. J Lab Clin Med. 2001 Feb;137(2):125-32. doi: 10.1067/mlc.2001.112235

9. Pohlers D, Brenmoehl J, Löffler I, Müller CK, Leipner C, Schultze-Mosgau S, Stallmach A, Kinne RW, Wolf G. TGF-beta and fibrosis in different organs molecular pathway imprints. Biochim Biophys Acta. 2009 Aug; 1792(8):746-56. doi: 10.1016/j.bbadis.2009.06.004

10. Fredriksson F, Christofferson RH, Lilja HE. Adhesive small bowel obstruction after laparotomy during infancy. Br J Surg. 2016 Feb;103(3):284-89. doi: $10.1002 /$ bjs. 10072

11. Филенко БП, Земляной ВП, Борсак ИИ, Иванов АС. Спаечная болезнь: профилактика и лечение. С-Петербург; 2013. 171 c. https://baltzdrav.ru/ images/files/borsak_monography.pdf

12. Behman R, Nathens AB, Byrne JP, Mason S, Look Hong N, Karanicolas PJ. Laparoscopic surgery for adhesive small bowel obstruction is associated with a higher risk of bowel injury: a population-based analysis of 8584 patients. Ann Surg. 2017 Sep;266(3):489-98. doi: $10.1097 /$ SLA.0000000000002369 
13. Thornblade LW, Truitt AR, Davidson GH, Flum DR, Lavallee DC. Surgeon attitudes and practice patterns in managing small bowel obstruction: a qualitative analysis. J Surg Res. 2017 Nov;219:347-53. doi: $10.1016 /$ j.jss.2017.06.052

14. Hajibandeh S, Hajibandeh S, Panda N, Khan RMA, Bandyopadhyay SK, Dalmia S, Malik S, Huq Z, Mansour M. Operative versus non-operative management of adhesive small bowel obstruction: A systematic review and meta-analysis. Int J Surg. 2017 Sep;45:58-66. doi: 10.1016/j.ijsu.2017.07.073

15. Lang J, Ma D, Xiang Y, Hua K, Liu K, Pan L, Wang P, Yao S, Zhao F, Cheng W, Cui M, Guo H, Guo R, Hong L, Li P, Liu M, Meng Y, Wang H, Wang J, Wang W, Wu M, Yang X, Zhang J. Chinese expert consensus on the prevention of abdominal pelvic adhesions after gynecological tumor surgeries. Ann Transl Med. 2020 Feb;8(4):79. doi: 10.21037/ atm.2020.02.53

16. Шальков ЮЛ. Спаечный синдром. Москва, РФ: БИНОМ; 2012. 238 с.

17. Симонян КС. Спаечная болезнь. Москва: Медицина; 1966. 250 с.

18. Напалков ПН. Спорное в абдоминальной спаечной болезни. В кн: Напалков ПН. Абдоминальная спаечная болезнь. Ленинград; 1977. с. 5-10.

19. Напалков ПН. Спорное во взглядах на абдоминальную болезнь. Вестн Хирургии им ИИ Грекова. 1977;(1):38-42.

20. Шальков ЮЛ. Дискуссионные аспекты спаечного синдрома. Вестн Хирургии им ИИ Грекова. 1996;(5):99-103.

21. Андреев АА, Остроушко АП, Кирьянова ДВ, Сотникова ЕС, Бритиков ВН. Спаечная болезнь брюшной полости. Вестн Эксперим и Клин Хирургии. 2017;10(4):320-26. doi: 10.18499/2070-478X2017-10-4-320-326

22. Catena F, Di Saverio S, Coccolini F, Ansaloni L, De Simone B, Sartelli M, Van Goor H. Adhesive small bowel adhesions obstruction: Evolutions in diagnosis, management and prevention. World J Gastrointest Surg. 2016 Mar 27;8(3):222-31. doi: 10.4240/wjgs.v8.i3.222

23. Женчевский РА. Спаечная болезнь. Москва: Медицина; 1999. 135 с.

24. Бондаревский ИЯ, Шалмагамбетов МС, Бордуновский ВН. Современное состояние проблемы прогнозирования и профилактики послеоперационного адгезиогенеза брюшины (обзор литературы). Урал Мед Журн. 2018;(1):69-78. https://www.elibrary. ru/item.asp?id=32400690

25. Bower KL, Lollar DI, Williams SL, Adkins FC, Luyimbazi DT, Bower CE. Small Bowel Obstruction. Surg Clin North Am. 2018 Oct;98(5):945-71. doi: 10.1016/j.suc.2018.05.007

26. Andrarawewa KL, Kirshner J, Mott JD, BarcellosHoff MH. TGF : roles in DNA responses. Cancer treatment and therapy. In: Jakowlew SB, editor. From transforming growth factor in cancer therapy (Vol. II). New York: Human Press; 2010. p. 321-31. https://www.springer.com/gp/book/9781588297150

27. Lebrun JJ. The dual role of TGF- in human cancer: from tumor suppression to cancer metastasis. ISRN Mol Biol. 2012;2012:381428. Published online 2012 Dec 24. doi: $10.5402 / 2012 / 381428$

28. Moustakas A, Heldin $\mathrm{CH}$. The regulation of TGFbeta signal transduction. Development. 2009 Nov;136(22):3699-14. doi: 10.1242/dev.030338

29. Young VJ, Ahmad SF, Duncan WC, Horne AW.
The role of TGF- in the pathophysiology of peritoneal endometriosis. Hum Reprod Update. 2017 Sep 1;23(5):548-59. doi: 10.1093/humupd/dmx016

30. Falk P, Angenete E, Bergström M, Ivarsson ML. TGF-1 promotes transition of mesothelial cells into fibroblast phenotype in response to peritoneal injury in a cell culture model. Int J Surg. 2013;11(9):977-82. doi: 10.1016/j.ijsu.2013.06.005

31. Jin X, Ren S, Macarak E, Rosenbloom J. Pathobiological mechanisms of peritoneal adhesions: The mesenchymal transition of rat peritoneal mesothelial cells induced by TGF-1 and IL-6 requires activation of Erk1/2 and Smad2 linker region phosphorylation. Matrix Biol. 2016 Apr;51:55-64. doi: 10.1016/j. matbio.2016.01.017

32. Liappas G, González-Mateo GT, Sánchez-Díaz R, Lazcano JJ, Lasarte S, Matesanz-Marín A, Zur R, Ferrantelli E, Ramírez LG, Aguilera A, FernándezRuiz E, Beelen RH, Selgas R, Sánchez-Madrid F, Martín P, Lypez-Cabrera M. Immune-Regulatory Molecule CD69 Controls Peritoneal Fibrosis. J Am Soc Nephrol. 2016 Dec;27(12):3561-76. doi: 10.1681/ ASN.2015080909

33. Jolly MK, Ware KE, Gilja S, Somarelli JA, Levine H. EMT and MET: necessary or permissive for metastasis? Mol Oncol. 2017 Jul;11(7):755-69. doi: 10.1002/1878-0261.12083

34. Loureiro J, Sandoval P, del Peso G, GynzalezMateo G, Fernández-Millara V, Santamaria B, Bajo MA, Sánchez-Tomero JA, Guerra-Azcona G, Selgas R, Lypez-Cabrera M, Aguilera AI. Tamoxifen ameliorates peritoneal membrane damage by blocking mesothelial to mesenchymal transition in peritoneal dialysis. PLoS One. 2013 Apr 23;8(4):e61165. doi: 10.1371/journal.pone.0061165. Print 2013.

35. Strippoli R, Moreno-Vicente R, Battistelli C, Cicchini C, Noce V, Amicone L, Marchetti A, Del Pozo MA, Tripodi M. Molecular Mechanisms Underlying Peritoneal EMT and Fibrosis. Stem Cells Int. 2016;2016:3543678. doi: 10.1155/2016/3543678

36. Назаренко АА, Акимов ВП, Малышкин ПО. Эпидемиология, патогенез и профилактика послеоперационного спаечного процесса в брюшной полости. Вестн. Хирургии им ИИ Грекова. 2016;175(5):114-18. https://www.vestnik-grekova.ru/ jour/article/view/359

37. Fan YH, Ji CX, Xu B, Fan HY, Cheng ZJ, Zhu $X G$. Long noncoding RNA activated by TGF- in human cancers: A meta-analysis. Clin Chim Acta. 2017 May;468:10-16. doi: 10.1016/j.cca.2017.02.001

38. Li DP, Fan J, Wu YJ, Xie YF, Zha JM, Zhou XM. MiR-155 up-regulated by TGF- promotes epithelial-mesenchymal transition, invasion and metastasis of human hepatocellular carcinoma cells in vitro. Am J Transl Res. 2017 Jun 15;9(6):2956-65. eCollection 2017. https://www.ncbi.nlm.nih.gov/pmc/ articles/PMC5489895/

39. Meng XM, Tang PM, Li J, Lan HY. TGF-/Smad signaling in renal fibrosis. Front Physiol. 2015 Mar 19;6:82. doi: 10.3389/fphys.2015.00082. eCollection 2015.

40. Salazar R, Roepman P, Willems SM, Brunen D, Kellner U, Midgley RA, Bernards Rene, Simon IM. Molecular subtyping of colorectal cancer to identify a mesenchymal tumor type that might benefit from TGF-beta pathway inhibition. J Clin Oncol. 2014;32(3 Suppl):456-56. https://ascopubs.org/doi/ abs/10.1200/jco.2014.32.3_suppl.456 
41. Шаповальянц СГ, Ларичев СЕ, Житарева ИВ, Бабкова ИВ, Сафаров АН. Современная диагностика странгуляционной формы острой спаечной тонкокишечной непроходимости. Вестн РГМУ. $2013 ;(1): 23-27$.

42. Шаповальянц СГ, Ларичев СЕ, Тимофеев МЕ. Лапароскопические вмешательства при острой спаечной тонкокишечной непроходимости. Эндоскоп Хирургия. 2014;(1):48-51.

43. Corcoran SE, O'Neill LA. HIF1 and metabolic reprogramming in inflammation. J Clin Invest. 2016 Oct 3;126(10):3699-707. doi: 10.1172/JCI84431

44. Haase VH. HIF-prolyl hydroxylases as therapeutic targets in erythropoiesis and iron metabolism. Hemodial Int. 2017 Jun;21(Suppl 1):S110-S124. doi: 10.1111/ hdi. 12567

45. Morishita Y, Ookawara S, Hirahara I, Muto S, Nagata D. HIF-1 mediates Hypoxia-induced epithelial-mesenchymal transition in peritoneal mesothelial cells. Ren Fail. 2016;38(2):282-89. doi: 10.3109/0886022X.2015.1127741

46. Wilson R. Changes in the coelomic microclimate during carbon dioxide laparoscopy: morphological and functional implications. Pleura Peritoneum. 2017 Mar 1;2(1):17-31. Published online 2017 Mar 17. doi: 10.1515/pp-pp-2017-0001

47. Byrne J, Saleh F, Ambrosini L, Quereshy F, Jackson TD, Okrainec A. Laparoscopic versus open surgical management of adhesive small bowel obstruction: a comparison of outcomes. Surg Endosc. 2015 Sep;29(9):2525-32. doi: 10.1007/s00464-0144015-7

48. Matsuzaki S, Vernis L, Bonnin M, Houlle C, Fournet-Fayard A, Rosano G, Lafaye AL, Chartier C, Barriere A, Storme B, Bazin JE, Canis M, Botchorishvilil R. Effects of low intraperitoneal pressure and a warmed, humidified carbon dioxide gas in laparoscopic surgery: a randomized clinical trial. Sci Rep. 2017;7:11287. Published online 2017 Sep 12. doi: 10.1038/s41598-017-10769-1

49. Kelly KN, Iannuzzi JC, Rickles AS, Garimella V, Monson JR, Fleming FJ. Laparotomy for small-bowel obstruction: first choice or last resort for adhesiolysis? A laparoscopic approach for small-bowel obstruction reduces 30-day complications. Surg Endosc. 2014 Jan;28(1):65-73. doi: 10.1007/s00464-013-3162-6

\section{REFERENCES}

1. Bello-Guerrero JA, Cruz-Santiago CA, LunaMartínez J. Pirfenidone vs. sodium hyaluronate/ carboxymethylcellulose as prevention of the formation of intra-abdominal adhesions after colonic surgery. A randomized study in an experimental model. Cir Esp. 2016 Jan;94(1):31-37. doi: 10.1016/j.ciresp.2015.06.005 2. Beyene RT, Kavalukas SL, Barbul A. Intra-abdominal adhesions: Anatomy, physiology, pathophysiology, and treatment. Curr Probl Surg. 2015 Jul;52(7):271-19. doi: 10.1067/j.cpsurg.2015.05.001

3. Skoglar A, Gunnarsson U, Falk P. Band adhesions not related to previous abdominal surgery - A retrospective cohort analysis of risk factors. Ann Med Surg (Lond). 2018 Nov 13;36:185-90. doi: 10.1016/j. amsu.2018.11.007. eCollection 2018 Dec.

4. Uslu Yuvaci H, Cevriolu AS, Gündüz Y, Akdemir N, Karacan A, Erkorkmaz Ü, Keskin A. Does applied ultrasound prior to laparoscopy predict the existence of intra-abdominal adhesions? Turk J Med Sci. 2020
Apr 9;50(2):304-11. doi: 10.3906/sag-1910-61. Online ahead of print

5. Lutsevich OE, Akimov VP, Shirinskii VG, Bichev AA. Voprosy patogeneza spaechnoi bolezni briushiny i sovremennye podkhody k ee preduprezhdeniiu: obzor literatury. Moscow Khirurg Zhurn. 2017;(3):11-26. https://www.mossj.ru/jour/article/view/22 (In Russ.) 6. Abrahams AC, Habib SM, Dendooven A, Riser BL, van der Veer JW, Toorop RJ, Betjes MG, Verhaar MC, Watson CJ, Nguyen TQ, Boer WH. Patients with encapsulating peritoneal sclerosis have increased peritoneal expression of connective tissue growth factor (CCN2), transforming growth factor-1, and vascular endothelial growth factor. PLoS One. 2014 Nov 10;9(11):e112050. doi: 10.1371/journal.pone.0112050. eCollection 2014.

7. Midgley AC, Rogers M, Hallett MB, Clayton A, Bowen T, Phillips AO, Steadman R. Transforming growth factor-1 (TGF-1)-stimulated fibroblast to myofibroblast differentiation is mediated by hyaluronan (HA)-facilitated epidermal growth factor receptor (EGFR) and CD44 co-localization in lipid rafts. J Biol Chem. 2013 May 24;288(21):14824-38. doi: 10.1074/ jbc.M113.451336

8. Zweers MM, Struijk DG, Smit W, Krediet RT. Vascular endothelial growth factor in peritoneal dialysis: a longitudinal follow-up. J Lab Clin Med. 2001 Feb;137(2):125-32. doi: 10.1067/mlc.2001.112235

9. Pohlers D, Brenmoehl J, Löffler I, Müller CK, Leipner C, Schultze-Mosgau S, Stallmach A, Kinne RW, Wolf G. TGF-beta and fibrosis in different organs - molecular pathway imprints. Biochim Biophys Acta. 2009 Aug;1792(8):746-56. doi: 10.1016/j. bbadis.2009.06.004

10. Fredriksson F, Christofferson RH, Lilja HE. Adhesive small bowel obstruction after laparotomy during infancy. Br J Surg. 2016 Feb;103(3):284-89. doi: $10.1002 /$ bjs. 10072

11. Filenko BP, Zemlianoi VP, Borsak II, Ivanov AS. Spaechnaia bolezn': profilaktika i lechenie. S-Peterburg; 2013. 171 p. https://baltzdrav.ru/images/ files/borsak_monography.pdf (In Russ.)

12. Behman R, Nathens AB, Byrne JP, Mason S, Look Hong N, Karanicolas PJ. Laparoscopic surgery for adhesive small bowel obstruction is associated with a higher risk of bowel injury: a population-based analysis of 8584 patients. Ann Surg. 2017 Sep;266(3):489-98. doi: 10.1097/SLA.0000000000002369

13. Thornblade LW, Truitt AR, Davidson GH, Flum DR, Lavallee DC. Surgeon attitudes and practice patterns in managing small bowel obstruction: a qualitative analysis. J Surg Res. 2017 Nov;219:347-53. doi: $10.1016 /$ j.jss.2017.06.052

14. Hajibandeh S, Hajibandeh S, Panda N, Khan RMA, Bandyopadhyay SK, Dalmia S, Malik S, Huq Z, Mansour M. Operative versus non-operative management of adhesive small bowel obstruction: A systematic review and meta-analysis. Int J Surg. 2017 Sep;45:58-66. doi: 10.1016/j.ijsu.2017.07.073

15. Lang J, Ma D, Xiang Y, Hua K, Liu K, Pan L, Wang P, Yao S, Zhao F, Cheng W, Cui M, Guo H, Guo R, Hong L, Li P, Liu M, Meng Y, Wang H, Wang J, Wang W, Wu M, Yang X, Zhang J. Chinese expert consensus on the prevention of abdominal pelvic adhesions after gynecological tumor surgeries. Ann Transl Med. 2020 Feb;8(4):79. doi: 10.21037/ atm.2020.02.53

16. Shal'kov IuL. Spaechnyi sindrom. Moscow, RF: 
BINOM; 2012. 238 p. (In Russ.)

17. Simonian KS. Spaechnaia bolezn'. Moscow: Meditsina; 1966. 250 p. (In Russ.)

18. Napalkov PN. Spornoe v abdominal'noi spaechnoi bolezni. Vkn: Napalkov PN. Abdominal'naia spaechnaia bolezn'. Leningrad; 1977. p. 5-10. (In Russ.)

19. Napalkov PN. Spornoe vo vzgliadakh na abdominal'nuiu bolezni. Vestn Khirurgii im II Grekova. 1977;(1):38-42. (In Russ.)

20.Shal'kov IuL. Diskussionnye aspekty spaechnogo sindroma. Vestn Khirurgii im II Grekova. 1996;(5):99103. (In Russ.)

21. Andreev AA, Ostroushko AP, Kiryanova DV, Sotnikova ES, Britikov VN. Adhesive disease of the abdominal cavity. J Exper and Clin Surg. 2017;10(4):32026. doi:10.18499/2070-478X2017-10-4-320-326 (In Russ.)

22. Catena F, Di Saverio S, Coccolini F, Ansaloni L, De Simone B, Sartelli M, Van Goor H. Adhesive small bowel adhesions obstruction: Evolutions in diagnosis, management and prevention. World J Gastrointest Surg. 2016 Mar 27;8(3):222-31. doi: 10.4240/wjgs.v8.i3.222

23 Zhenchevskii RA. Spaechnaia bolezn'. Moscow: Meditsina; 1999. 135 p. (In Russ.)

24. Bondarevsky IYa, Shalmagambetov MS, Bordunovsky VN. Pathophysiology and prevention of postoperative peritoneal adhesions (review article).Ural Med Zhurn. 2018;(1):69-78. https://www.elibrary.ru/ item.asp?id $=32400690$ (In Russ.)

25. Bower KL, Lollar DI, Williams SL, Adkins FC, Luyimbazi DT, Bower CE. Small Bowel Obstruction. Surg Clin North Am. 2018 Oct;98(5):945-71. doi: 10.1016/j.suc.2018.05.007

26. Andrarawewa KL, Kirshner J, Mott JD, BarcellosHoff MH. TGF : roles in DNA responses. Cancer treatment and therapy. In: Jakowlew SB, editor. From transforming growth factor in cancer therapy (Vol. II). New York: Human Press; 2010. p. 321-31. https://www.springer.com/gp/book/9781588297150

27. Lebrun JJ. The dual role of TGF- in human cancer: from tumor suppression to cancer metastasis. ISRN Mol Biol. 2012;2012:381428. Published online 2012 Dec 24. doi: $10.5402 / 2012 / 381428$

28. Moustakas A, Heldin $\mathrm{CH}$. The regulation of TGFbeta signal transduction. Development. 2009 Nov;136(22):3699-14. doi: 10.1242/dev.030338

29. Young VJ, Ahmad SF, Duncan WC, Horne AW. The role of TGF- in the pathophysiology of peritoneal endometriosis. Hum Reprod Update. 2017 Sep 1;23(5):548-59. doi: 10.1093/humupd/dmx016

30. Falk P, Angenete E, Bergström M, Ivarsson ML.

TGF-1 promotes transition of mesothelial cells into fibroblast phenotype in response to peritoneal injury in a cell culture model. Int J Surg. 2013;11(9):977-82. doi: 10.1016/j.ijsu.2013.06.005

31. Jin X, Ren S, Macarak E, Rosenbloom J. Pathobiological mechanisms of peritoneal adhesions: The mesenchymal transition of rat peritoneal mesothelial cells induced by TGF-1 and IL-6 requires activation of Erk1/2 and Smad2 linker region phosphorylation. Matrix Biol. 2016 Apr;51:55-64. doi: 10.1016/j. matbio.2016.01.017

32. Liappas G, González-Mateo GT, Sánchez-Díaz R, Lazcano JJ, Lasarte S, Matesanz-Marín A, Zur R, Ferrantelli E, Ramírez LG, Aguilera A, FernándezRuiz E, Beelen RH, Selgas R, Sánchez-Madrid F, Martín P, Lypez-Cabrera M. Immune-Regulatory Molecule CD69 Controls Peritoneal Fibrosis. J Am
Soc Nephrol. 2016 Dec;27(12):3561-76. doi: 10.1681/ ASN.2015080909

33. Jolly MK, Ware KE, Gilja S, Somarelli JA, Levine H. EMT and MET: necessary or permissive for metastasis? Mol Oncol. 2017 Jul;11(7):755-69. doi: 10.1002/1878-0261.12083

34. Loureiro J, Sandoval P, del Peso G, GynzalezMateo G, Fernández-Millara V, Santamaria B, Bajo MA, Sánchez-Tomero JA, Guerra-Azcona G, Selgas R, Lypez-Cabrera M, Aguilera AI. Tamoxifen ameliorates peritoneal membrane damage by blocking mesothelial to mesenchymal transition in peritoneal dialysis. PLoS One. 2013 Apr 23;8(4):e61165. doi: 10.1371/journal.pone.0061165. Print 2013.

35. Strippoli R, Moreno-Vicente R, Battistelli C, Cicchini C, Noce V, Amicone L, Marchetti A, Del Pozo MA, Tripodi M. Molecular Mechanisms Underlying Peritoneal EMT and Fibrosis. Stem Cells Int. 2016;2016:3543678. doi: 10.1155/2016/3543678

36. Nazarenko AA, Akimov VP, Malyshkin PO. Epidemiologiia, patogenez i profilaktika posleoperatsionogo spaechnogo protsessa $\mathrm{v}$ briushnoi polosti. Vestn. Khirurgii im II Grekova. 2016;175(5):11418. https://www.vestnik-grekova.ru/jour/article/ view/359 (In Russ.)

37. Fan YH, Ji CX, Xu B, Fan HY, Cheng ZJ, Zhu $\mathrm{XG}$. Long noncoding RNA activated by TGF- in human cancers: A meta-analysis. Clin Chim Acta. 2017 May;468:10-16. doi: 10.1016/j.cca.2017.02.001

38. Li DP, Fan J, Wu YJ, Xie YF, Zha JM, Zhou XM. MiR-155 up-regulated by TGF- promotes epithelial-mesenchymal transition, invasion and metastasis of human hepatocellular carcinoma cells in vitro. Am J Transl Res. 2017 Jun 15;9(6):2956-65. eCollection 2017. https://www.ncbi.nlm.nih.gov/pmc/ articles/PMC5489895/

39. Meng XM, Tang PM, Li J, Lan HY. TGF-/Smad signaling in renal fibrosis. Front Physiol. 2015 Mar 19;6:82. doi: 10.3389/fphys.2015.00082. eCollection 2015.

40. Salazar R, Roepman P, Willems SM, Brunen D, Kellner U, Midgley RA, Bernards Rene, Simon IM. Molecular subtyping of colorectal cancer to identify a mesenchymal tumor type that might benefit from TGF-beta pathway inhibition. J Clin Oncol. 2014;32(3 Suppl):456-56. https://ascopubs.org/doi/abs/10.1200/ jco.2014.32.3_suppl.456

41. Shapovalyants SG, Larichev SE, Zhitareva IV, Babkova IV, Safarov AN. Modern diagnostics of strangulated form of acute adhesive small bowel obstruction. Вестн РГМУ. 2013;(1):23-27. (In Russ.) 42. Shapoval'iants SG, Larichev SE, Timofeev ME. Laparoskopicheskie vmeshatel'stva pri ostroi spaechnoi tonkokishechnoi neprokhodimosti. Endoskop Khirurgiia. 2014;(1):48-51.

43. Corcoran SE, O'Neill LA. HIF1 and metabolic reprogramming in inflammation. J Clin Invest. 2016 Oct 3;126(10):3699-707. doi: 10.1172/JCI84431

44. Haase VH. HIF-prolyl hydroxylases as therapeutic targets in erythropoiesis and iron metabolism. Hemodial Int. 2017 Jun;21(Suppl 1):S110-S124. doi: 10.1111/ hdi. 12567

45. Morishita Y, Ookawara S, Hirahara I, Muto S, Nagata D. HIF-1 mediates Hypoxia-induced epithelial-mesenchymal transition in peritoneal mesothelial cells. Ren Fail. 2016;38(2):282-89. doi: 10.3109/0886022X.2015.1127741

46. Wilson R. Changes in the coelomic microclimate 
during carbon dioxide laparoscopy: morphological and functional implications. Pleura Peritoneum. 2017 Mar 1;2(1):17-31. Published online 2017 Mar 17. doi: $10.1515 /$ pp-pp-2017-0001

47. Byrne J, Saleh F, Ambrosini L, Quereshy F, Jackson TD, Okrainec A. Laparoscopic versus open surgical management of adhesive small bowel obstruction: a comparison of outcomes. Surg Endosc. 2015 Sep;29(9):2525-32. doi: 10.1007/s00464-0144015-7

48. Matsuzaki S, Vernis L, Bonnin M, Houlle C, Fournet-Fayard A, Rosano G, Lafaye AL, Chartier

\section{Адрес для корреспонденции}

220116, Республика Беларусь,

г. Минск, пр. Дзержинского, д. 83,

Белорусский государственный медицинский

университет, кафедра военно-полевой хирургии, тел.: +375 29 377-96-67,

e-mail: mdkluiko@gmail.com,

Клюйко Дмитрий Александрович

\section{Сведения об авторах}

Клюйко Дмитрий Александрович, к.м.н., доцент, полковник м/с, начальник кафедры военно-полевой хирургии военно-медицинского института, Белорусский государственный медицинский университет, г. Минск, Республика Беларусь. http://orcid.org/0000-0003-4090-500X

\section{Информация о статье}

Поступила 18 января 2021 г.

Принята в печать 27 сентября 2021 г. Доступна на сайте 1 ноября 20212.
C, Barriere A, Storme B, Bazin JE, Canis M, Botchorishvili1 R. Effects of low intraperitoneal pressure and a warmed, humidified carbon dioxide gas in laparoscopic surgery: a randomized clinical trial. Sci Rep. 2017;7:11287. Published online 2017 Sep 12. doi: 10.1038/s41598-017-10769-1

49.Kelly KN, Iannuzzi JC, Rickles AS, Garimella V, Monson JR, Fleming FJ. Laparotomy for small-bowel obstruction: first choice or last resort for adhesiolysis? A laparoscopic approach for small-bowel obstruction reduces 30-day complications. Surg Endosc. 2014 Jan;28(1):65-73. doi: 10.1007/s00464-013-3162-6

\section{Address for correspondence}

220116, Republic of Belarus,

Minsk, Dzerzhinsky Avenue, 83,

Belarusian State Medical University,

the Department of Military Field Surgery,

+375 29 377-96-67

e-mail: mdkluiko@gmail.com

Klyuiko Dmitry A.

\section{Information about the authors}

Klyuiko Dmitry A., PhD, Associate Professor, Medical Colonel, Head of the Department of Military Field Surgery, Belarusian State Medical University, Minsk, Republic of Belarus.

http://orcid.org/0000-0003-4090-500X

\section{Article history}

Arrived: 18 January 2021

Accepted for publication: 27 September 2021

Available online: 1 January 2022 